\title{
The Expanding Role of Lower Fidelity Simulation Technologies in Aviation Training
}

\author{
D.R. Farrow, Instructional Systems Development Specialist \\ Advanced Qualification Program Branch \\ Federal Aviation Administration \\ Washington, DC 20041-2027 USA
}

Keywords: Aircraft Crew Training, Aircraft Display Human Factors, Air Transportation, Simulation, Simulation Software, Training.

\section{INTRODUCTION}

This paper describes the basic approach used by the Federal Aviation Administration (FAA) to classify the various levels of simulation devices used for training and certifying pilots within the airline industry. It also discusses these devices in terms of the differing types of training and evaluation (certification) activities most appropriate to each. Because of the similarities between the aviation and nuclear power industries (government regulation, safety orientation, high-technology operations, team performance, etc.) many of the training and evaluation concepts utilized by the aviation industry should generalize well to the nuclear power industry. This paper also discusses the projects in progress at the FAA to accommodate the use of lower levels of simulation fidelity in training and evaluation applications. It concludes with a discussion of the human factors issues that currently limit more effective utilization of the current inventory of FAA approved devices.

\section{LEVELS OF FLIGHT SIMULATION EQUIPMENT}

\section{A. Aviation Training Basics}

This paper focuses on the functional characteristics and related training strategies associated with the FAA-approved simulation devices used in the training and certification of commercial airline pilots. Although occasional reference will be made to the training needs of the private, general aviation pilot, most FAA flight training equipment guidance has been developed for the pilots of air transport category aircraft.

There are two major categories of aircraft training at most airlines: initial equipment training and recurrent training. Initial equipment training certifies a pilot to fly a particular type of aircraft, such as a Boeing 737 . Recurrent training, which occurs every six or twelve months, re-certifies that pilot to continue to fly that aircraft. The certification requirements for these courses are defined in terms of a series of qualification events, consisting of flight maneuvers and procedures performed under normal, abnormal and emergency conditions. These qualification events are documented in a series of FAA regulations.

\section{B. Defining Levels of Fidelity for Flight Training Equipment}

The FAA has; over time, evolved a systematic process for classifying training equipment according to its level of fidelity, and indicating those training and certification activities that may be accomplished, for FAA credit, in each level of equipment $[1,2,3]$. The FAA identifies as a Level $D$ simulator a device that may completely replace the aircraft for all initial and recurrent training and certification requirements. (These requirements are defined in terms of approximately 70 separate qualification events). This full fidelity device is defined in terms of its performance characteristics, functional characteristics and other features. 
After defining full flight training equipment, the FAA goes on to define 10 progressively less capable categories of training equipment in terms of their own performance and functional characteristics. These 10 classifications of lower fidelity devices are framed as levels. The full taxonomy includes 11 levels.

Levels 1-7 are referred to as Level 1-7 Flight Training Devices (FTD's), while levels 8-11 are referred to as Level A-D simulators.

These 11 categories of devices are then matched against the 70 qualification events in a matrix. This matrix indicates which of the 70 qualification events may be trained or evaluated in each device type. The net result of this system is to provide the FAA with (1) a standardized definition of full fidelity flight training equipment (the Level D simulator), as well as 10 discrete categories of equipment with progressively less robust fidelity, and (2) guidance as to which training and certification events are appropriate for each category of equipment.

\section{Functional Description of a Full Flight Simulator}

The FAA provides a functional description of each of these 11 levels of flight training equipment. This abbreviated description of a Level D full flight simulator provides one example. Other definitions may be found in $[4,5]$. Level D flight simulators have the following characteristics: (1) Systems representations, switches, and controls which are required by the type design of the aircraft and by the user's training program. (2) Systems which respond appropriately and accurately to the switches and controls of the aircraft being simulated. (3) Full-scale replication of the cockpit of the aircraft being simulated. (4) Correct simulation of the aerodynamic characteristics including ground effect, and ground dynamic characteristics of the aircraft being simulated. (5) Correct simulation of selected environmentally affected aerodynamic and ground dynamic characteristics of the aircraft being simulated considering the full range of its flight envelope in all approved configurations. (6) Correct and realistic simulation of the effects of environmental conditions which the aircraft might encounter. (7) Control forces, control dynamics, and control travel which correspond to the aircraft. (8) Instructor controls and seat. (9) A daylight, dusk and night visual system with at least a 75 degree horizontal by 30 degree vertical field of view for each pilot station. (10) A motion system with at least six degrees of freedom. The other 10 levels are similarly described along these same dimensions.

\section{Taxonomy of Simulation Devices for Flight Training}

A full flight commercial aircraft simulator consists of three basic components: An enclosed cockpit, a visual system, and a motion system, all three interfaced to provide a realistic synthetic flight environment.

The FAA has, for the purpose of this taxonomy, defined as a simulator that training equipment that contains all three components. It has defined as a flight training device that equipment that includes a cockpit representation, may include visual system, and does not include a motion system. The functional distinction is that FTD's can be used for training and certifying ground operation tasks, inflight operation tasks and some non-visual approach tasks, but cannot be used for takeoff tasks, inflight maneuvering tasks, visual approach tasks or landing tasks. It is important to note that this limited definition of simulators is for administrative purposes only, and that the research literature on simulators applies to equipment the FAA labels FTD's as well as it applies to equipment the FAA labels simulators. For the purposes of this paper, all FTS's should be considered lower fidelity simulation devices.

The devices in the FAA taxonomy may be described in decreasing order of fidelity, such that each device has less physical and functional fidelity, as well as less training application, than the device above it in the taxonomy. For example, a Level D simulator has a night, dusk and daylight visual system, a Level $\mathrm{C}$ simulator has only a night and dusk visual system, and a Level B simulator has only a night visual system. A Level $\mathrm{C}$ simulator has a 70 degree field of view and a motion base with six degrees of freedom, while a Level B simulator has only a 45 degree field of view and a motion base with only three degrees of freedom. The aerodynamic modeling for a Level 7 FTD must be "correct" relative to the aircraft, for a Level 6 FTD this 
modeling need only "closely represent" the aircraft's characteristics, and for a Level 5 FTD the simulated aerodynamics need only be "representative" of the aircraft.

This taxonomy was not developed a priori based on any systematic theory of simulator design for flight training and evaluation. It was, instead, developed a posteriori based on a substantial inventory of devices already in operation in the field. For this reason, there are of necessity a number of gaps and inconsistencies in functionality across the taxonomy. This occasionally uneven graduation in progressive device features is reflected in the uneven progression in the percent of the 70 qualification events that can be accomplished for credit in each. For example, a Level 4 FDT can be used to evaluate only $10 \%$ of these events, a Level 5 can be used for $20 \%$, a Level 6 or 7 for $40 \%$, a Level A for $65 \%$, a Level B for $70 \%$, and a level $\mathrm{C}$ or $\mathrm{D}$ for close to $100 \%$ [6]. These numbers, like most the numbers in this paper, are approximate and are used to simplify complex issues that cannot be addressed here, but which are covered in detail in the references.

\section{TRAINING IMPLICATIONS}

\section{A. General Training Significance of the Levels of Devices}

These increasingly complex devices also support, in general, an increasingly complex series of training activities. For example, a Level 4 FTD supports learning, development, and practice of skills and cockpit procedures necessary to understand and operate the integrated systems of a specified aircraft. A Level 5 FTD supports the learning, development, and practice of skills, cockpit procedures, and instrument flight procedures necessary to understand and operate the integrated systems of a specific aircraft in typical flight operations in real time. A Level 6 FTD supports the learning, development, and practice of skills in cockpit procedures, instrument flight maneuvers, certain symmetrical maneuvers and flight characteristics necessary to operate the integrated systems of a specific aircraft in typical flight operations in real time.

This classification scheme does, therefore, support a protocol whereby students begin training in lower level devices, and migrate to higher level devices over time as their skills and knowledge increase.

\section{B. Approaches to Fidelity}

The research literature identifies a substantial number of different types of fidelity measures that may be used to develop synthetic task environments: physical, functional, task, behavioral, psychological, equipment, etc. [7]. As illustrated above, the FAA system focuses on equipment, physical, task and functional fidelity. When classifying training equipment, the FAA conducts a series of performance tests, which are objective engineering evaluations of the simulator's aerodynamic and ground handling characteristics as compared to those of the aircraft. They also perform a series of functional tests, which are subjective assessments by expert pilots who determine whether the "feel" of the simulator's controls, the responsiveness of the motion system, and the cues from the visual system provide sufficiently realistic feedback to the pilot for each of the 70 qualification events. Other FAA guidance [8] specifies that variations from the standard device-by -qualificationevents matrix described above must be justified by the submission of a cueing analysis [9] and a cockpit features analysis. The basic approach is to match the requirements of the qualification event against the features of the training equipment to indicate which events are supported by which equipment.

The current FAA approach provides a conservative and reliable set of measures of fidelity, focusing on the classic measurement of features and functions. It has not, as yet, incorporated the more recent dimensions of what has been identified as psychological, or cognitive fidelity. This construct has been defined as the realism of the information content, presentation, and management options that is present in a simulated task environment [10]. While the physical fidelity approach relies on the surface properties of a situation, the cognitive approach is based on the deep structure of the task in terms of its processing, response and resource demands [11]. The incorporation of this additional dimension of fidelity into the specification of both qualification events and simulator characteristics has the potential for significant improvements in pilot training and evaluation, based on the increasing importance of cognitive 
skill training and evaluation within the aviation community.

\section{Crew Resource Managment (CRM) Skill Development}

Studies by the National Aeronautics and Space Administration (NASA) and the National Transportation Safety Board (NTSB) have indicated that over $60 \%$ of all fatal air carrier accidents are related to human factors problems in the cockpit [12], now commonly referred to as CRM issues. CRM skills are broadly defined to include (1) communications processes and decision behavior, (2) team building and maintenance, and (3) workload management and situational awareness [13]. In response to these findings, the FAA has recently mandated CRM training for all airline pilots. Training in the traditional technical skills of maneuvers and procedures must be enhanced with considerations for these newly mandated CRM skills. But what training and evaluation activities are most appropriate for these skills, and in what type of training device? Is full physical fidelity required, or even desired, for training and evaluating these cognitive skills?

Aviation training has historically focused more on psychomotor skill training (so-called technical or "stick and rudder" skills) than on cognitive skill training (decision-making skills). The FAA's initial reaction to the need for additional focus on CRM was to introduce classroom training in generic decision-making skills. Over the years, this was augmented with training exercises in FTD's and simulators that included both technical and cognitive skills. These scenario-based exercises were called line oriented flight training (LOFT), and brought decision-making training into the simulated cockpit. Later still, these scenarios were also incorporated into evaluations, known as line oriented evaluations (LOE's). The industry has now developed a sophisticated scenariodevelopment methodology to support these LOFT's and LOE's [14], which maximizes their utility within the training syllabus.

The strength of these scenarios is that they integrate the technical and cognitive elements of each flightcrew task. The possible weakness is that this methodology uses the level of fidelity required by the technical task most closely associated with each cognitive task in order to determine the fidelity requirements of the training scenario. This conservative approach has required the use of high levels of physical fidelity for the development of cognitive skills, when the use of such complex and expensive equipment may not be required, or even desired. This is particularly acute when remediation is prescribed. When a pilot fails a scenario in a full flight simulator, and that failure is based primarily on a cognitive skill deficiency, current practice requires training and re-evaluation in that same full flight simulator. Yet some studies suggest that the use of lower fidelity devices may be more training and cost effective for such remediation [15].

\section{ONGOING EFFORTS IN LOWER FIDELITY TRAINING AND EVALUATION}

The FAA is a safety organization, and as such has set very high standards for the physical and functional fidelity requirements of the flight training equipment it approves for each pilot qualification event. It is also a dynamic organization, however, and is open to adjusting standards as devices improve, as new scientific information becomes available, and as the industry gains experience with simulation. There are at present a number of projects ongoing that reflect an increasing level of comfort on the part of the FAA with increased training and evaluation in lower fidelity devices.

\section{A. Increased Use of Lower Fidelity Devices for Evaluation}

The FAA recently increased the range of evaluation permitted in Level $\mathrm{C}$ simulators. While the FAA has always permitted all evaluations for all crewmembers to be performed in a Level $D$ simulator, it has traditionally limited the use of Level $\mathrm{C}$ simulator evaluations by first officers (as opposed to captains). But the FAA has recently allowed first officers to complete additional categories of evaluations in Level $\mathrm{C}$ simulators. Changes in the use of Level C simulators are particularly important, as the number of Level $C$ simulators currently deployed exceeds the total number of all level A, $B$ and D simulators combined.

The FAA is also considering changes to the qualification standards (functional description) 
of the Level B simulator. Because recurrent training and checking dominate all airlines' training budgets, and because the FAA allows for $100 \%$ of recurrent training and evaluation to be completed in a Level B simulator, the FAA has set about to examine the requirements for Level B simulators. The goal of the project is to determine whether recent improvements in simulation technology, particularly aerodynamic modeling and motion cueing, can justify changes in standards (limits, tolerances and motion cueing) that will preserve the training effectiveness of the device, but lower the cost. A lower cost will have the effect of increasing the availability of this level of device within the airline industry.

\section{B. Increased Flexibility in the Deployment of Simulation Technology}

The Advanced Qualification Program (AQP) is a voluntary regulatory option that provides air carriers, manufacturers and training centers with additional flexibility in the use of simulators, FTD's and other simulation technology. This program allows participants to modify, with FAA approval, not only the level of training equipment assigned to current qualification events, but also the listing of qualification events itself. This program was designed to encourage innovation in aviation training, and as such holds the most promise for innovation in the use of lower level simulation technologies.

C. Development of Formal FAA Guidance for the Use of Personal Computer-Based Aviation Training Devices (PCTAD's)

The FAA has monitored the research regarding the training effectiveness of aviation training devices based on personal computers for many years, as well as sponsoring its own research in this area [16]. As a result of the effort, the FAA has drafted formal guidance (in the form of an upcoming FAA Advisory Circular) on the use of these devices within the scope of FAA-approved training programs. This guidance is to be issued by the branch of the FAA that provides oversight to general aviation aircraft, rather than the branch that provides guidance to air carrier aircraft. This suggests that the FAA currently views this technology, in its present form, as more applicable to pilots who are in the earlier stages of aviation training.

\section{CONCLUSIONS}

The FAA has developed a systematic process for classifying various levels of simulation equipment, and for matching each level with the qualification events for which each device is most appropriate. They have also developed programs and criteria for re-evaluating both the levels and the events. At this time, most of the program work within the FAA is aimed an relaxing these original standards, as changes in technology, research and accumulated industry experience justify their reconsideration.

\section{REFERENCES}

[1,4] Federal Aviation Administration, Airplane Simulator Qualification (Advisory Circular 120 . 40B), Washington, DC: Department of Transportation, 1991.

[2,5] Federal Aviation Administration, Airplane Flight Training Device Qualification (Advisory Circular 120-45A), Washington, DC: Department of Transportation, 1992.

[3,6,8] Federal Aviation Administration, Advanced Qualification Program(Advisory Circular 12054), Washington, DC: Department of Transportation, 1991.

[7] D.H. Andrews, L.A. Carroll and H.H. Bell, "The future of selective fidelity in training devices," Proceedings of the 1994 Interservice/Industry Training Systems and Education Conference, pp. 3-12.

[9] Federal Aviation Administration, A Systematic Determination of Skill and Simulator Requirements for Airline Pilot Certification, Washington, DC: Department of Transportation, 1985.

[10] J.S. Bresee and W.W. Wagner, "Cognitive fidelity and training device design," Proceedings of the Royal Aeronautical Society Conference on Part Task Trainers, 1993. pp 16.1-16.6.

[11,15] D. Gopher, M. Weil and T. Bareket, "Transfer of skill from a computer game trainer to flight," Human Factors, vol. 36, no. 3, September 1994, pp. 387-405.

[12] Federal Aviation Administration, Advanced Qualification Program; Final Rule, Washington, DC: Department of Transportation, 1990.

[13] Federal Aviation Administration, Crew Resource Management (Advisory Circular 120-51B, Change 1), Washington, DC: Department of Transportation, 1995. 
Airline Transport Association, Line Operational Simulations: LOFT Scenario Design, Conduct and Validation, Washington, DC: Airline

Transport Association, 1994.

[16] Williams, K. W. (Ed.), Summary Proceedings of the Joint Industry-FAA Conference on the Development and Use of PC-Based Aviation Training Devices, Oklahoma City, OK: Civil Aeromedical Institute, 1994.

\section{BIOGRAPHY}

Douglas Farrow is an Instructional Systems Development Specialist with the Advanced Qualification Program Branch of the Federal Aviation Administration, located at Dulles International Airport in Chantilly, Virginia. He provides guidance to Advanced Qualification Program airlines, aircraft manufacturers and training centers on the design, development, delivery and evaluation of their aircrew training programs. He received his $\mathrm{Ph}$. D. in Instructional Systems from Florida State University in 1982, and has developed pilot training programs for numerous military and civilian aircraft since that time. Dr. Farrow has authored over 100 individual lessons and over 50 professional papers, reports and presentations.

Disclaimer: The views presented in this paper represent those of the author and do not necessarily reflect those of the Federal Aviation Administration. 